\title{
Copper nanoclusters: Synthesis, characterization and properties
}

\author{
LU YiZhong ${ }^{1,2}$, WEI WenTao ${ }^{1,3} \&$ CHEN Wei ${ }^{1 *}$ \\ ${ }^{1}$ State key Laboratory of Electroanalytical Chemistry, Changchun Institute of Applied Chemistry, Chinese Academy of Sciences, Changchun \\ 130022, China; \\ ${ }^{2}$ Graduate School of the Chinese Academy of Sciences, Beijing 100049, China; \\ ${ }^{3}$ School of Chemistry and Environmental Engineering, Changchun University of Science and Technology, Changchun 130022, China
}

Received July 11, 2011; accepted September 14, 2011

\begin{abstract}
Metal nanoclusters with a core size smaller than $2 \mathrm{~nm}$ have attracted much attention because of their unique physical and chemical properties. Among the studied metal nanoclusters, gold and silver have been studied extensively by size-controlled synthesis, structural characterization and properties investigations. Recently, considerable research effort has been devoted to the investigation of copper nanoclusters. In this review, we highlight recent progress in the study of copper nanoclusters in terms of synthesis methods, characterization techniques and their novel optical and catalytic properties.
\end{abstract}

copper, nanoclusters, fluorescence, electrocatalyst, nanomaterials, synthesis

Citation: $\quad$ Lu Y Z, Wei W T, Chen W. Copper nanoclusters: Synthesis, characterization and properties. Chin Sci Bull, 2012, 57: 41-47, doi: 10.1007/s11434-0114896-y

Metal nanoclusters have attracted considerable attention because of their unique size-dependent properties such as photoluminescence [1], intrinsic magnetism [2], electrochemical characteristics [3], chirality [4] and catalysis [5], which deviate significantly from those of their corresponding bulk metals and large nanoparticles. Metal nanoclusters with a core size comparable to the Fermi wavelength of an electron bridge the gap between molecules and nanoparticles. In the past decade, noble metal nanoclusters (mainly $\mathrm{Au}$ and $\mathrm{Ag}$ ) have been extensively synthesized and studied using alkanethiols and other ligands as protecting agents by taking advantage of the strong affinity of thiol, amido or phosphine groups to noble metal surfaces and their excellent stability in different media [6-8]. Copper is widely used in industry because of its high conductivity, similar properties to gold and silver and much lower cost. However, compared to the extensive studies on gold and silver, reports on copper nanoclusters are still scarce primarily because of their susceptibility to oxidation and the difficulty in preparing extremely tiny particles. Recently, several methods have been successfully developed to synthesize tiny copper

*Corresponding author (email: weichen@ciac.jl.cn) nanoclusters and monolayer-protected clusters with unique optical and catalytic properties [9-14]. In this review, we will initially focus on the synthesis techniques that have been used for copper nanocluster preparation such as template-based syntheses, electrochemical methods, water-in-oil (w/o) microemulsion techniques, the typical Brust-Schiffrin method and microwave-assisted polyol synthesis. We then summarize the often used techniques for the structural and optical characterizations of copper nanoclusters such as UV-Vis spectroscopy, transmission electron microscopy (TEM) and high-resolution TEM techniques, powder X-ray diffraction analysis, X-ray photoelectron spectroscopy, FT-IR measurements, energy dispersive X-ray (EDX) analysis and mass spectrometry etc. We also discuss the properties of copper nanoclusters that have recently been discovered. Finally, we give some concluding remarks and the outlook for research into copper nanoclusters in the near future.

\section{Synthesis of copper nanoclusters}

Several synthesis methods have been reported for copper nanocluster preparation. In this section, we summarize all 
the methods that have been shown to be effective for the synthesis of copper nanoclusters. These methods include template-based synthesis, electrochemical techniques, the water-in-oil (w/o) microemulsion strategy, the typical Brust-Schiffrin method and microwave-assisted polyol synthesis method.

\subsection{Template-based synthesis}

Over the past few decades, template-based synthesis techniques have been shown to be efficient synthesis methods for the preparation of metal nanoparticles. The metal nanoparticles form within the interiors of templates such as molecular sieves, polymers, polyelectrolytes and dendrimers. An advantage of this method is that the core size and the size distribution of the metal nanoparticles can be controlled by templates with different cavity dimensions. Crooks and co-workers [9] prepared $\mathrm{Cu}$ nanoclusters within "nanoreactors" of hydroxyl terminated, fourth-generation polyamidoamine dendrimers (PAMAM $(\mathrm{G} 4-\mathrm{OH})$ ). In this method the dendrimer serves as a synthetic template and it also provides a ligand field for copper clusters to avoid aggregation. The PAMAM dendrimer has a uniform structure containing interior tertiary amines that can form coordination bonds with transition metal ions. In this report, PAMAM and $\mathrm{CuSO}_{4}$ were co-dissolved so that the $\mathrm{Cu}^{2+}$ ions could be extracted into the interior of PAMAM. $\mathrm{Cu}^{2+}$ ions were located in the $\mathrm{G} 4-\mathrm{OH}$ dendrimers $\left(\mathrm{G} 4-\mathrm{OH} / \mathrm{Cu}^{2+}\right)$ and were reduced to metallic copper nanoclusters by $\mathrm{NaBH}_{4}$. However, because $\mathrm{H}^{+}$could effectively compete with $\mathrm{Cu}^{2+}$ for binding with the tertiary amine sites it is necessary to adjust the $\mathrm{pH}$ of the solution to an appropriate value during the synthesis process.

Almost at the same time, Balogh and Tomalia [10] synthesized stable copper nanoclusters with a PAMAM dendrimer as a template. The UV-visible absorption spectra indicated that very tiny copper clusters were formed in the dendrimer.

\subsection{Electrochemical synthesis method}

The electrochemical synthesis method, first developed by Reetz et al. in 1994 [15] is a very promising technique for the production of metal nanoparticles because of their simplicity and their versatile application in the preparation of particles with different shapes and sizes. With this method, a sacrificial anode is used as the metal source. The metal ions produced by anodic dissolution are reduced at the cathodic surface to form metallic particles, which are stabilized by surfactants in the solution. Based on this mechanism, Vilar-Vidal et al. [12] successfully synthesized stable and photoluminescent small $\mathrm{Cu}_{n}(n \leqslant 14)$ nanoclusters in an aqueous solution. In a typical synthesis, copper ions were produced during the electrolysis of the $\mathrm{Cu}$ anode in the presence of a $0.1 \mathrm{~mol} / \mathrm{L}$ tetrabutylammonium nitrate aqueous solution as a supporting electrolyte, a platinum sheet as the cathode and $\mathrm{Ag} / \mathrm{AgCl}$ as the reference electrode, respectively. The prepared copper nanoclusters are soluble in nonpolar solvents and exhibit strong photoluminescence with large bandgaps. From the TEM measurement shown in Figure 1, the average cluster size was determined to be 0.61 $\pm 0.13 \mathrm{~nm}$. Importantly, the clusters showed high stability over years at low temperature $\left(\sim 4^{\circ} \mathrm{C}\right)$. With this method, clusters with high purity in addition to precise particle-size control could be easily achieved by adjusting the current density.

\subsection{Water-in-oil (w/o) microemulsion technique}

A microemulsion, the liquid mixture of aqueous and organic phases, provides an ideal chemical reactor at the nanoscale level and has been widely used to prepare metallic nanoparticles with the precise control of particle size, shape and narrow size distributions [16]. Recently, Vazquez-Vazquez et al. [11] reported the preparation of a series of small atomic copper clusters by the microemulsion technique. At the same time, they found that the core size of the copper clusters can be easily controlled by adjusting the amount of reducing agent used for the synthesis. In their report, the microemulsion system was initially formed by mixing high purity sodium dodecyl sulfate (SDS, as a surfactant), isopentanol (as a cosurfactant), cyclohexane (as an oily phase) and $\mathrm{Cu}$ (II) sulfate solution (as an aqueous phase). An appropriate amount of freshly prepared $\mathrm{NaBH}_{4}$ aqueous solution $(2 \%(\mathrm{w} / \mathrm{v}))$ was then slowly added dropwise into the formed water-in-oil microemulsion. Moreover, copper nanoclusters with different sizes were obtained when using different percentages of $\mathrm{NaBH}_{4}$. For example, very tiny copper nanoclusters, $\mathrm{Cu}_{n}(n \leqslant 13)$ were synthesized when the percentage of $\mathrm{NaBH}_{4}$ was very low $(<10 \%)$. Nanoparticles of $\sim 3 \mathrm{~nm}$ in size showed the typical copper surface plasmonic

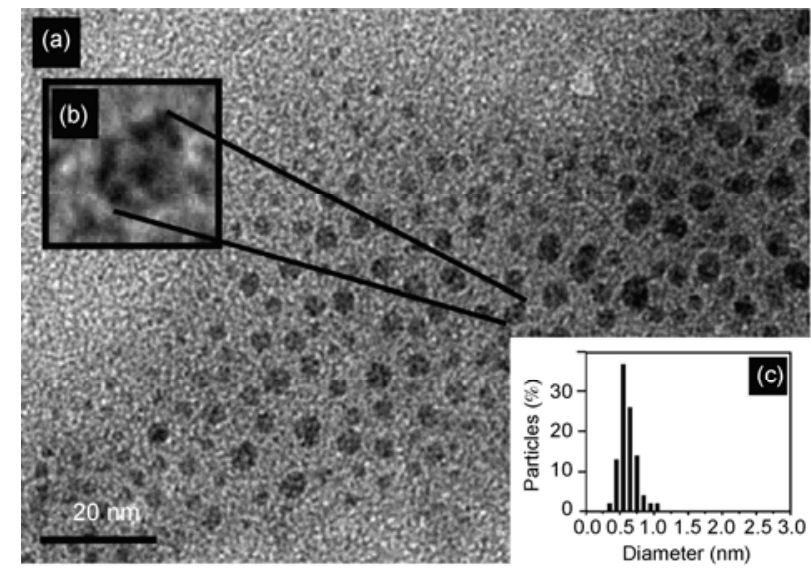

Figure 1 (a) TEM image of copper nanoclusters synthesized by an electrochemical technique. (b) Enlargement of one $\mathrm{Cu}$ cluster aggregate. (c) Size histogram of individual clusters from the aggregates shown in (a) with an average cluster size of $0.61 \pm 0.13 \mathrm{~nm}$. Reprinted from ref. [12] with permission from the American Chemical Society. 
band and were obtained when the near stoichiometric amounts of reducing agent reached $60 \%$. They also found that the photoluminescence properties of the copper nanoclusters are strongly dependent on the core size. Fluorescence with blue emission was only found for clusters of $\mathrm{Cu}_{n}$ at $n \leqslant 13$. Figure 2 shows the evolution of copper cluster size with an increase in the percentage of $\mathrm{NaBH}_{4}(\alpha)$ and their optical properties are dependent on the cluster size.

\subsection{Modified Brust-Schiffrin method}

Since gold nanoparticles were first synthesized using a two-phase method by Brust and co-workers in 1994 [17], the so-called Brust-Schiffrin method has been widely used to prepare monolayer-protected metal nanoclusters because of its simple operation and the highly stable obtained nanoclusters. Two processes, the formation of the metallic nanoclusters and the simultaneous passivation of the metal core by the protection of ligand monolayers are supposed to be involved in Brust-Schiffrin method. In the synthesis process, the metal ions initially transfer from the aqueous phase to the organic phase with the assistance of phasetransfer agents (eq. (1)) followed by a metal ions reduction process in the presence of reducing agents (eq. (2)):

$$
\mathrm{AuCl}_{4}^{-}(\mathrm{aq})+\mathrm{N}\left(\mathrm{C}_{8} \mathrm{H}_{17}\right)_{4}^{+}(\text {toluene }) \rightarrow \underset{\text { (toluene })}{\mathrm{N}\left(\mathrm{C}_{8} \mathrm{H}_{17}\right)_{4}^{+} \mathrm{AuC1}_{4}^{-}}
$$

$$
\begin{gathered}
m \mathrm{AuCl}_{4}^{-} \text {(toluene) }+n \mathrm{C}_{12} \mathrm{H}_{25} \mathrm{SH} \text { (toluene) }+3 m \mathrm{e}^{-} \\
\rightarrow 4 m \mathrm{Cl}^{-}(\mathrm{aq})+\left(\mathrm{Au}_{m}\right) \mathrm{C}_{12}\left(\mathrm{H}_{25} \mathrm{SH}\right)_{n} \text { (toluene) }
\end{gathered}
$$

Recently, Goulet and Lennox [18] reported the identification and quantification of precursor species of $\mathrm{Au}, \mathrm{Ag}$ and $\mathrm{Cu}$ nanoclusters based on ${ }^{1} \mathrm{H}$ NMR analysis and they proposed a new formation mechanism (eq. (3)):

$$
\left[\mathrm{NR}_{4}\right]\left[\mathrm{AuX} \mathrm{X}_{4}\right]+2 \mathrm{R}^{\prime} \mathrm{SH} \rightarrow\left[\mathrm{NR}_{4}\right]\left[\mathrm{AuX}_{2}\right]+\mathrm{R}^{\prime} \mathrm{SSR}+2 \mathrm{HX}
$$

They proposed that before the addition of the reductant
$\mathrm{NaBH}_{4}$ the metal precursor is the TOA-metal (I) halide complex [TOA] $\left[\mathrm{AuX}_{2}\right]$ rather than the $[\text { metal(I)SR }]_{n}$-like polymers, which have been generally recognized as precursors in previous reports. Such a mechanism was also confirmed by $\mathrm{Li}$ and co-workers [19]. The revised viewpoint could help to understand the synthetic mechanism and also help in the synthesis of other size-controlled metal nanoclusters.

Inspired by the revised synthetic mechanism, we have successfully synthesized stable $\mathrm{Cu}_{n}(n \leqslant 8)$ nanoclusters by a simple one-pot wet chemical reduction method using 2-mercapto-5- $n$-propylpyrimidine (MPP) as the protecting ligand based on a revised Brust-Schiffrin method [13]. The $[\mathrm{TOA}]_{2}\left[\mathrm{CuBr}_{4}\right]$ complex was synthesized according to a modified literature approach [20]. After the complex was dissolved in ethanol it was cooled to $0^{\circ} \mathrm{C}$ in an ice bath and 5 equivalents of MPP $(\mathrm{MPP} / \mathrm{Cu}$ salt $=5 / 1)$ was added with vigorous stirring. The mixture was left to react for $6 \mathrm{~h}$ before the addition of sodium borohydride. Copper nanoclusters were obtained after $7 \mathrm{~h}$ of reaction. The as-synthesized $\mathrm{Cu}_{n}$ nanoclusters were found to only be soluble in nonpolar solvents such as toluene, hexane and chloroform.

\subsection{Microwave-assisted polyol synthesis}

More recently, Kawasaki et al. [14] reported the synthesis of fluorescent $\mathrm{Cu}$ nanoclusters via a microwave-assisted polyol method without using additional protective and reducing agents. In a typical synthesis, the solution of $\mathrm{CuCl}_{2}$ in ethylene glycol was mixed with $0.5 \mathrm{~mol} / \mathrm{L} \mathrm{NaOH}$ in ethylene glycol in a glass tube by magnetic stirring for $10 \mathrm{~min}$. The solution mixture was then placed in a microwave oven and was vigorously stirred for $30 \mathrm{~min}$ at a reaction temperature of $185^{\circ} \mathrm{C}$ under a $\mathrm{N}_{2}$ atmosphere. After the color of the solution changed to light brown, $1 \mathrm{~mol} / \mathrm{L} \mathrm{HCl}$ aqueous solution was added. The obtained $\mathrm{Cu}$ nanoclusters were extracted into a diethyl ether phase and dried using a rotary evaporator.

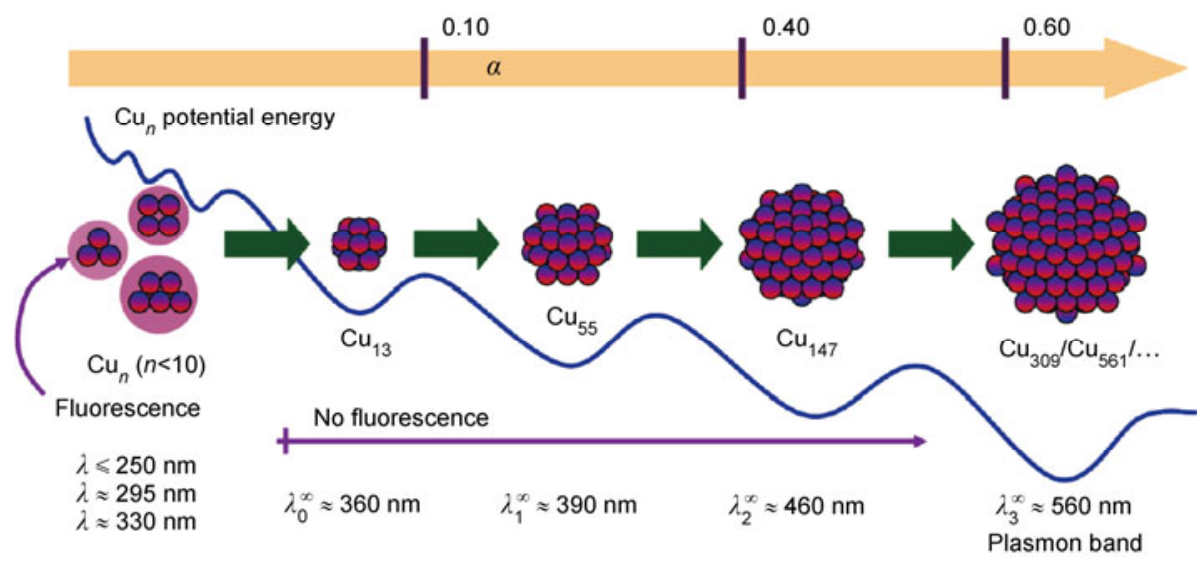

Figure 2 (Color online) Schematic of the evolution of copper cluster size with an increase in the percentage of $\mathrm{NaBH}_{4}(\alpha)$. Copper clusters, $\mathrm{Cu}_{n}$, with $n \leqslant$ 13, show fluorescence. Estimated wavelengths of the plasmon band for different sized copper clusters are shown at the bottom. Reprinted from ref. [11] with permission of the American Chemical Society. 
Note that the $\mathrm{Cu}$ nanoclusters synthesized by the microwave-assisted polyol method were highly resistant to oxidation and exhibited strong fluorescence. This method provides a new route for the synthesis of other metal clusters without the addition of protecting ligands.

As discussed above, each method has its own advantages for the synthesis of copper nanoclusters. However, despite all these efforts devoted to the synthesis of copper nanoclusters, more studies are needed for the synthesis of high yield, high purity and water-soluble copper nanoclusters with a high quantum yield of fluorescence.

\section{Characterization of copper nanoclusters}

Various analysis techniques have been used for the structural, optical and catalytic characterization of the synthesized copper nanoclusters. Among the applied techniques TEM, HRTEM and mass spectrometry are often used to study the size, size distribution, shape and molecular formula of the copper nanoclusters. UV-Vis and fluorescence spectroscopy have been used to study optical properties. XPS, FT-IR, EDX are also often used to obtain information about the oxidation state of copper, the surface structure and the elemental distribution of the clusters. Several important characterization techniques will be discussed in detail below.

\subsection{UV-Vis spectroscopy}

Because of the quantum confinement effect of ultrasmall copper nanoclusters, molecular-like discrete electronic structures have optical properties that are significantly different from their larger counterparts. Important information about the obtained copper nanoclusters can be derived from $\mathrm{UV}-\mathrm{V}$ is absorption spectra. UV-Vis spectroscopy has been recognized as a powerful tool for qualitative identification during the formation of copper nanoclusters.

\subsection{Transmission electron microscopy (TEM) or high- resolution TEM (HRTEM) techniques}

TEM or HRTEM are widely used to determine the core diameter and shape of $\mathrm{Cu}$ nanoclusters. A dilute solution of $\mathrm{Cu}$ clusters is usually used to prepare TEM or HRTEM samples to avoid cluster aggregation during TEM measurements. Although TEM and HRTEM are conventional and powerful techniques to characterize the size and crystal structure of nanomaterials they are not reliable techniques for the evaluation of cluster size distribution for copper nanoclusters because of their extremely small size.

\subsection{X-ray photoelectron spectroscopy}

X-ray photoelectron spectroscopy (XPS) has been used to evaluate the chemical states of the metal cores in nano- material studies. For instance, the oxidation state of copper nanoclusters synthesized by a wet chemical reduction method was investigated by XPS measurements (Figure 3). The XPS spectrum shows two peaks at 932.3 and $952.0 \mathrm{eV}$, which could be assigned to the binding energy of $\mathrm{Cu} 2 \mathrm{p}_{3 / 2}$ and $\mathrm{Cu} 2 \mathrm{p}_{1 / 2}$ electrons of metallic copper $(\mathrm{Cu}(0))$, respectively. The absence of an XPS signal from $\mathrm{Cu}$ (II) suggests the lack of significant oxidation in the $\mathrm{Cu}$ nanoclusters. However, for as-synthesized copper nanoclusters prepared by the electrochemical technique [12], binding energy peaks from both $\mathrm{Cu}(0)$ and $\mathrm{Cu}$ (II) are present in the XPS spectra (Figure 4). After $\mathrm{Ar}^{+}$ion sputtering treatment $\mathrm{Cu}$ (II) can be removed completely and only $\mathrm{Cu}(0)$ and a small amount of $\mathrm{Cu}$ (I) remains in the clusters. Such results suggest that the copper nanoclusters synthesized by different techniques may have different oxidation states, which can be detected reliably with XPS measurements.

\subsection{FT-IR characterization}

To obtain stable and mono-dispersed metal nanoclusters,

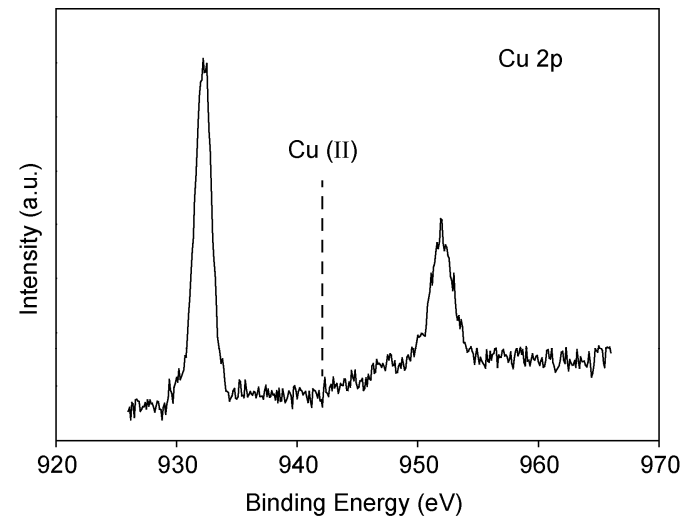

Figure 3 X-ray photoelectron spectrum of $\mathrm{Cu} 2 \mathrm{p}$ electrons in $\mathrm{Cu}$ nanoclusters synthesized by wet chemical reduction. The dashed line shows the binding energy position of $\mathrm{Cu}$ (II) $2 \mathrm{p}$ electrons. Reprinted from ref. [13] with permission of the American Chemical Society.

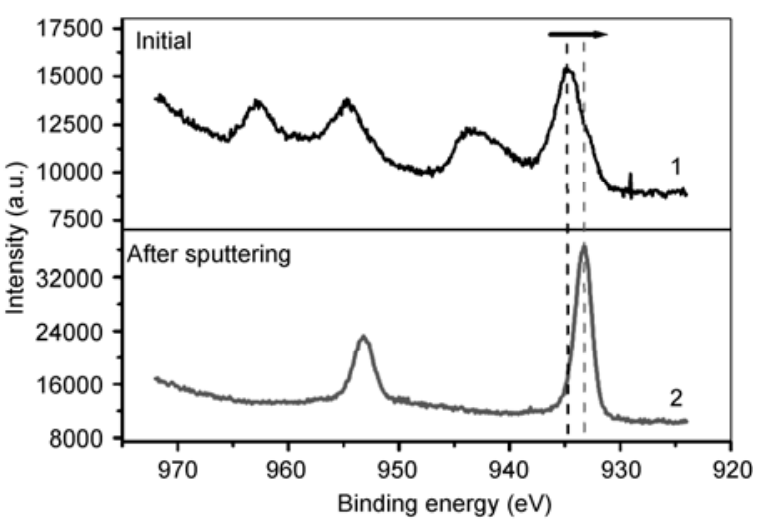

Figure 4 X-ray photoelectron spectra of copper clusters obtained by an electrochemical technique before (line 1) and after sputtering with $\mathrm{Ar}^{+}$ions (line 2). Reprinted from ref. [12] with permission of the American Chemical Society. 
stabilizers are usually used to protect the cluster surface and thus prevent aggregation. FT-IR spectroscopy can be used to study the formation mechanism and the surface chemical environment of metal clusters. Figure 5 shows the FT-IR spectra of the monomeric 2-mercapto-5- $n$-propylpyrimidine (MPP) and MPP-protected copper nanoclusters [13]. The broad peak at $2525 \mathrm{~cm}^{-1}$, which corresponds to the S-H stretching vibration mode in MPP molecules disappears in the $\mathrm{Cu}$-MPP clusters indicating that MPP molecules are bound onto the copper surface through $\mathrm{Cu}-\mathrm{S}$ bonds. Combined with XPS data, it can be concluded that copper nanoclusters were synthesized successfully using MPP as protecting ligands.

\subsection{Mass spectrometry (MS)}

As described above, TEM and HRTEM with currently available resolutions are not reliable in the determination of the precise molecule formula of very tiny metal nanoclusters. In almost all metal nanocluster studies, mass spectrometry is used to analyze the chemical composition of the clusters. Recently, various types of MS techniques such as laser desorption/ionization mass spectrometry (LDI-MS) [21], laser desorption/ionization time-of-flight (LDI-TOF) [22], matrix-assisted laser desorption ionization mass spectrometry (MALDI-MS) [5,23] and electrospray ionization mass spectrometry (ESI-MS) in positive- or negative-ion modes $[13,24,25]$ have been applied to the study of metal nanoclusters. For example, ESI-MS in positive-ion mode was used in our study on copper nanoclusters [13]. From the ESI-MS spectrum shown in Figure 6, the highest mass peak, $m / z \approx 1120$, could be assigned to the $\mathrm{Cu}$ clusters with a composition of $\mathrm{Cu}_{8} \mathrm{~L}_{4}\left(\mathrm{~L}=\mathrm{C}_{7} \mathrm{H}_{9} \mathrm{~N}_{2} \mathrm{~S}\right)$ whereas those in the lower mass range might be because of the different fragments as labeled in Figure 6. From the MS result, $\mathrm{Cu}_{8}$ clusters were found to be the dominant $\mathrm{Cu}$-containing components in the synthesized product. LDI-TOF mass spectroscopy showed that $\mathrm{Cu}_{n}$ with $n \leqslant 14$ were synthesized using

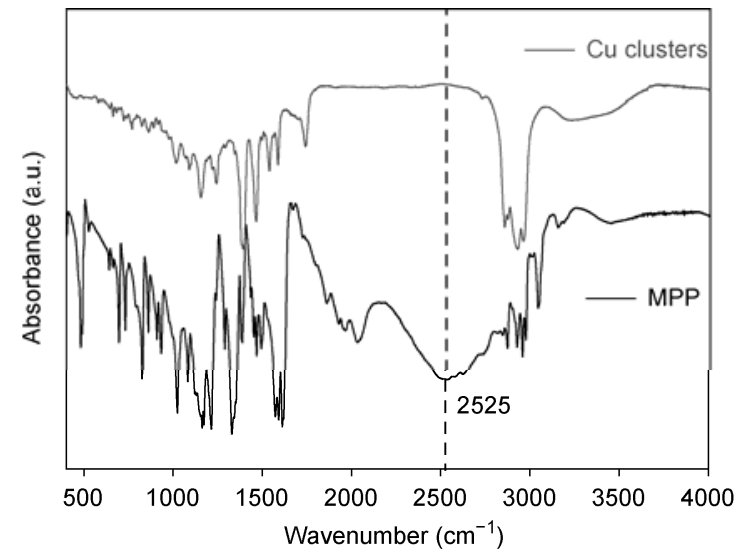

Figure 5 FT-IR spectra of the MPP-protected $\mathrm{Cu}$ nanoclusters and monomeric MPP (2-mercapto-5- $n$-propylpyrimidine). Reprinted from ref. [13] with permission of the American Chemical Society.

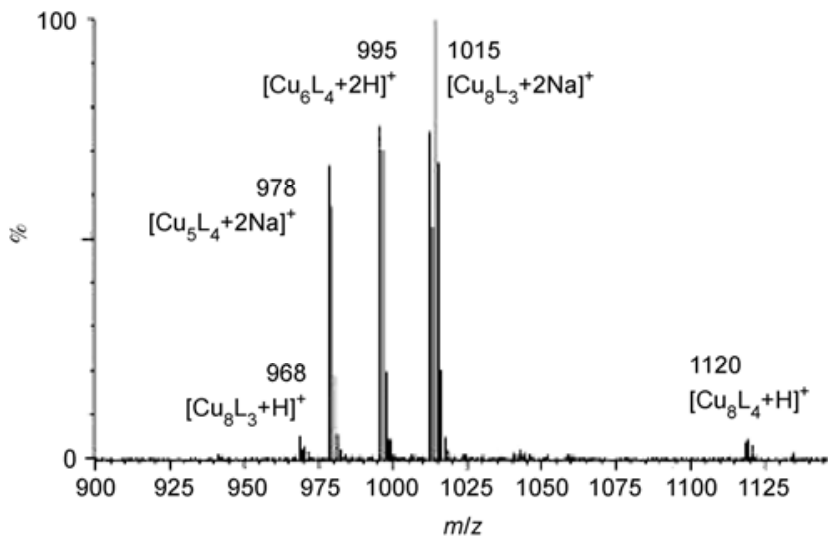

Figure 6 ESI mass spectrum of a copper cluster sample detected in positive-ion mode. Reprinted from ref. [13] with permission of the American Chemical Society.

an electrochemical technique [12].

Practical research usually requires the combined use of characterization techniques such as those mentioned above to obtain complete information about synthesized copper nanoclusters.

\section{Properties of copper nanoclusters}

Because of their small size and special discrete energy levels as well as bandgap energy structures, metal nanoclusters exhibit unique electronic, optical and catalytic properties by comparison with larger nanoparticles. These properties include strong photoluminescence [6,7], high (electro) catalytic activities [5,26], quantized capacitance charging (or single electron transfer) [27-29]. These metal nanoclusters with novel properties show great potential applications in fluorescence analysis, chemical/biosensors, optoelectronic devices and biological labeling etc. [30-31]. The optical (UV-Vis absorption and fluorescence) and catalytic properties of copper nanoclusters have mainly been studied over the last few years.

\subsection{Multiband UV-Vis absorption}

Characteristic surface plasmon resonance (SPR) peaks are usually observed for large metal nanoparticles during UV-Vis absorption measurements. For instance, particle size-dependent SPR peaks of $\mathrm{Au}, \mathrm{Ag}$ and $\mathrm{Cu}$ can be observed at around 520, 420 and $600 \mathrm{~nm}$, respectively. However, metal nanoclusters exhibit molecular-like HOMOLUMO electronic features and multiband step-wise optical absorption behavior instead of collective plasmon excitation [32], which is due to the loss of their metallic nature as a result of the quantum confinement effect. Figure 7 shows the UV-Vis absorption spectrum of MPP-capped $\mathrm{Cu}_{n}(n \leqslant 8)$ nanoclusters [13]. Compared to the UV-Vis absorption of monomeric MPP, three well-resolved absorption peaks at 


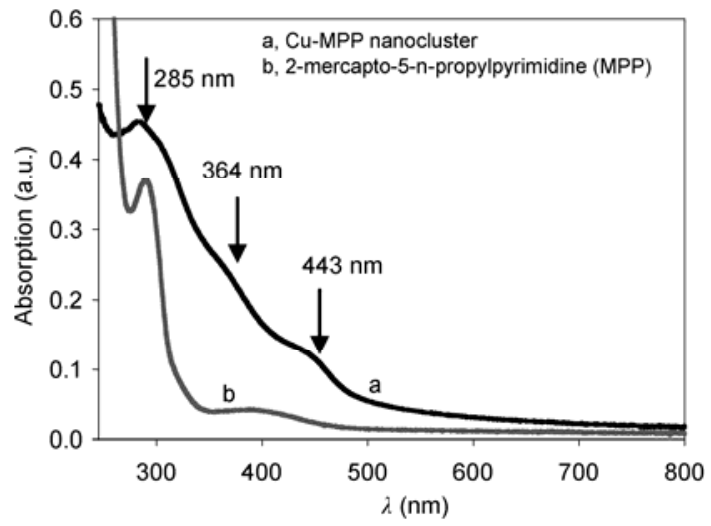

Figure 7 UV-Vis absorption spectra of the 2-mercapto-5-n-propylpyrimidine (MPP) capped $\mathrm{Cu}$ nanoclusters and monomeric MPP. Reprinted from ref. [13] with permission of the American Chemical Society.

285,364 , and $443 \mathrm{~nm}$ are present in the spectrum of the clusters. This multiple band optical absorption is due to interband electronic transitions of the $\mathrm{Cu}$ clusters from discrete energy levels.

\subsection{Photoluminescent properties}

Because of an interband transition and an intraband HOMOLUMO transition, metal clusters can exhibit photoluminescence properties. For example, $\mathrm{Cu}$ nanoclusters obtained via microwave-assisted polyol synthesis show blue emission at $475 \mathrm{~nm}$ under $350 \mathrm{~nm}$ excitation (Figure 8) [14]. For $\mathrm{Cu}_{n}(n$ $\leqslant 8$ ) clusters synthesized by wet chemical reduction, unique dual emissions at 423 and $593 \mathrm{~nm}$ were observed [13]. As has been observed for gold nanoclusters and complexes the emission at $423 \mathrm{~nm}$ could be a result of the interband transition from excited states in the sp band to the $\mathrm{d}$ band and the emission at $593 \mathrm{~nm}$ could be a result of the intraband HOMO-LUMO transition within the sp band. These novel

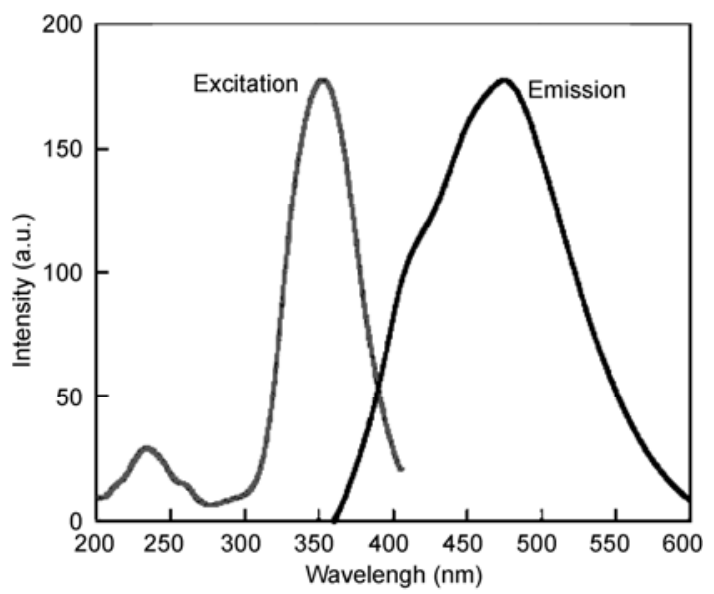

Figure 8 Excitation ( $\lambda_{\mathrm{em}}=475 \mathrm{~nm}$ ) and emission $\left(\lambda_{\mathrm{ex}}=355 \mathrm{~nm}\right)$ spectra of $\mathrm{Cu}$ nanoclusters obtained by the microwave-assisted polyol synthesis method. Reprinted from ref. [14] with permission of the Royal Society of Chemistry. fluorescence properties make the metal nanoclusters ideal fluorophores for applications in bioimaging, biolabeling and fluorescence analysis etc. For instance, Guo et al. [33] developed a method for the selective detection of $\mathrm{Hg}^{2+}$ by using oligonucleotide-protected silver nanoclusters as fluorescent probes. In their study, high sensitivity with the detection limit of $5 \mathrm{nmol} / \mathrm{L}$ and high selectivity for $\mathrm{Hg}^{2+}$ from other metal ions was successfully realized. Compared to toxic semiconductor quantum dots and expensive $\mathrm{Au}$ and Ag nanoclusters, the cheaper and nontoxic fluorescent copper nanoclusters will be more widely applied in fluorescence-related fields.

\subsection{Catalytic properties}

Previous studies have shown that nanometer-sized gold clusters exhibit high catalytic and electrocatalytic activities compared to the inert bulk gold metal $[5,26]$. Recently, oxygen electrochemical reduction has been studied using a series of gold nanoclusters with sizes smaller than $2 \mathrm{~nm}(0.8$ to $1.7 \mathrm{~nm}$ ) [5]. The Au nanoclusters exhibited much higher electrocatalytic activity for oxygen reduction and higher catalytic performance was obtained for the smaller clusters. Such enhanced catalytic activity may be in part because of the large fraction of surface $\mathrm{Au}$ atoms with low coordination numbers. Moreover, theoretical studies have shown that by decreasing the core size of the Au nanoclusters the $\mathrm{d}$ bands become narrower and shift towards the Fermi level, which will favor $\mathrm{O}_{2}$ adsorption by ultrasmall clusters and will further benefit the ORR. The electrocatalytic activity towards the oxygen reduction reaction (ORR) was also studied using copper and silver nanoclusters [13,34]. The results showed that $\mathrm{Cu}_{n}(n \leqslant 8)$ and $\mathrm{Ag}_{7}$ nanoclusters exhibit high catalytic activity for the electro-reduction of oxygen. The onset potential of oxygen reduction $(-0.07 \mathrm{~V}$ vs $\mathrm{Ag} /$ $\mathrm{AgCl})$ on the copper nanoclusters was found to be highly comparable to that observed with $\mathrm{Au}_{11}$ clusters $(-0.08 \mathrm{~V}$ vs $\mathrm{Ag} / \mathrm{AgCl}$ ) and some commercial Pt catalysts, which suggests that copper nanoclusters might serve as effective platinum-free electrocatalysts for fuel cells.

\section{Conclusion and remarks}

In summary, we provide an overview of the synthesis, characterization and properties of copper nanoclusters that have been reported over the past few years. Much effort has been put into copper nanoclusters research and several efficient synthetic techniques have been developed to prepare copper nanoclusters. With evidence from electron microscopy and mass spectrometry, stable copper nanoclusters smaller than $\mathrm{Cu}_{13}$ and $\mathrm{Cu}_{8}$ have been successfully synthesized by electrochemical and wet chemical reduction methods, respectively. The synthesized copper nanoclusters exhibit unique fluorescent and catalytic properties. Despite 
the recent progress in this field, much research work is still required into copper nanoclusters. Since hydrophobic $\mathrm{Cu}$ nanoclusters with mixed core sizes have usually synthesized in previous studies, obtaining monodispersed and stable water-soluble copper nanoclusters is a very important issue for their application in fluorescence analysis and cellular biology.

This work was supported by the National Natural Science Foundation of China (21043013) and the Startup Funds for Scientific Research, Changchun Institute of Applied Chemistry, Chinese Academy of Sciences.

$1 \mathrm{Xu} \mathrm{H} \mathrm{X,} \mathrm{Suslick} \mathrm{K} \mathrm{S.} \mathrm{Sonochemical} \mathrm{synthesis} \mathrm{of} \mathrm{highly} \mathrm{fluorescent}$ Ag nanoclusters. ACS Nano, 2010, 4: 3209-3214

2 González B S, Rodríguez M J, Blanco C, et al. One step synthesis of the smallest photoluminescent and paramagnetic PVP-protected gold atomic clusters. Nano Lett, 2010, 10: 4217-4221

3 Ingram R S, Hostetler M J, Murray R W, et al. 28 kDa alkanethiolate-protected $\mathrm{Au}$ clusters give analogous solution electrochemistry and STM coulomb staircases. J Am Chem Soc, 1997, 119: 92799280

4 Cathcart N, Kitaev V. Silver nanoclusters: Single-stage scaleable synthesis of monodisperse species and their chirooptical properties. J Phys Chem C, 2010, 114: 16010-16017

5 Chen W, Chen S W. Oxygen electroreduction catalyzed by gold nanoclusters: Strong core size effects. Angew Chem Int Ed, 2009, 48: 4386-4389

6 Diez I, Ras R H A. Fluorescent silver nanoclusters. Nanoscale, 2011, 3: $1963-1970$

7 Jin R C. Quantum sized, thiolate-protected gold nanoclusters. Nanoscale, 2010, 2: 343-362

8 Murray R W. Nanoelectrochemistry: Metal nanoparticles, nanoelectrodes, and nanopores. Chemical Rev, 2008, 108: 2688-2720

9 Zhao M Q, Sun L, Crooks R M. Preparation of $\mathrm{Cu}$ nanoclusters within dendrimer templates. J Am Chem Soc, 1998, 120: 4877-4878

10 Balogh L, Tomalia D A. Poly (amidoamine) dendrimer-templated nanocomposites. 1. Synthesis of zerovalent copper nanoclusters. J Am Chem Soc, 1998, 120: 7355-7356

11 Vazquez-Vazquez C, Banobre-Lopez M, Mitra A, et al. Synthesis of small atomic copper clusters in microemulsions. Langmuir, 2009, 25 : 8208-8216

12 Vilar-Vidal N, Blanco M C, Lopez-Quintela M A, et al. Electrochemical synthesis of very stable photoluminescent copper clusters. J Phys Chem C, 2010, 114: 15924-15930

13 Wei W T, Lu Y Z, Chen W, et al. One-pot synthesis, photoluminescence, and electrocatalytic properties of subnanometer-sized copper clusters. J Am Chem Soc, 2011, 133: 2060-2063

14 Kawasaki H, Kosaka Y, Myoujin Y, et al. Microwave-assisted polyol synthesis of copper nanocrystals without using additional protective agents. Chem Commun, 2011, 47: 7740-7742

15 Reetz M T, Helbig W. Size-selective synthesis of nanostructured transition metal clusters. J Am Chem Soc, 1994, 116: 7401-7402

16 Lopez-Quintela M A, Tojo C, Blanco M C, et al. Microemulsion dy- namics and reactions in microemulsions. Curr Opin Colloid Interf Sci, 2004, 9: 264-278

17 Brust M, Walker M, Bethell D, et al. Synthesis of thiol-derivatised gold nanoparticles in a two-phase liquid-liquid system. J Chem Soc Chem Commun, 1994, 801-802

18 Goulet P J G, Lennox R B. New insights into Brust-Schiffrin metal nanoparticle synthesis. J Am Chem Soc, 2010, 132: 9582-9584

19 Li Y, Zaluzhna O, Xu B L, et al. Mechanistic insights into the BrustSchiffrin two-phase synthesis of organo-chalcogenate-protected metal nanoparticles. J Am Chem Soc, 2011, 133: 2092-2095

20 Gill N S, Taylor F B, Hatfield W E, et al. Tetrahalo complexes of dipositive metals in the first transition series. Inorg Synth, 1967, 9: 136-142

21 Alvarez M M, Khoury J T, Schaaff T G, et al. Optical absorption spectra of nanocrystal gold molecules. J Phys Chem B, 1997, 101: 3706-3712

22 Waters C A, Mills A J, Johnson K A, et al. Purification of dodecanethiol derivatised gold nanoparticles. Chem Commun, 2003, 39: 540-541

23 Dass A, Stevenson A, Dubay G R, et al. Nanoparticle MALDI-TOF mass spectrometry without fragmentation: $\mathrm{Au}_{25}\left(\mathrm{SCH}_{2} \mathrm{CH}_{2} \mathrm{Ph}\right)_{18}$ and mixed monolayer $\mathrm{Au}_{25}\left(\mathrm{SCH}_{2} \mathrm{CH}_{2} \mathrm{Ph}\right)_{18-x} \mathrm{~L}_{x}$. J Am Chem Soc, 2008, 130: 5940-5946

24 Wu Z K, Lanni E, Chen W Q, et al. High yield, large scale synthesis of thiolate-protected $\mathrm{Ag}_{7}$ clusters. J Am Chem Soc, 2009, 131: 16672-16673

25 Zheng J, Petty J T, Dickson R M. High quantum yield blue emission from water-soluble $\mathrm{Au}_{8}$ nanodots. J Am Chem Soc, 2003, 125: 7780-7781

26 Herzing A A, Kiely C J, Carley A F, et al. Identification of active gold nanoclusters on iron oxide supports for $\mathrm{CO}$ oxidation. Science, 2008, 321: 1331-1335

27 Chen S W, Ingram R S, Hostetler M J, et al. Gold nanoelectrodes of varied size: Transition to molecule-like charging. Science, 1998, 280: 2098-2101

28 Chen W, Davies J R, Ghosh D, et al. Carbene-functionalized ruthenium nanoparticles. Chem Mater, 2006, 18: 5253-5259

29 Chen W, Ghosh D, Sun J, et al. Dithiocarbamate-protected ruthenium nanoparticles: synthesis, spectroscopy, electrochemistry and STM studies. Electrochim Acta, 2007, 53: 1150-1156

30 Wang H H, Lin C A J, Lee C H, et al. Fluorescent gold nanoclusters as a biocompatible marker for in vitro and in vivo tracking of endothelial cells. ACS Nano, 2011, 5: 4337-4344

31 Adhikari B, Banerjee A. Facile synthesis of water-soluble fluorescent silver nanoclusters and Hg-II sensing. Chem Mater, 2010, 22: 4364-4371

32 Bakr O M, Amendola V, Aikens C M, et al. Silver nanoparticles with broad multiband linear optical absorption. Angew Chem Int Ed, 2009 , 48: 5921-5926

33 Guo W W, Yuan J P, Wang E K. Oligonucleotide-stabilized Ag nanoclusters as novel fluorescence probes for the highly selective and sensitive detection of the $\mathrm{Hg}^{2+}$ ion. Chem Commun, 2009, 23: 33953397

34 Lu Y Z, Chen W. Size effect of silver nanoclusters on their catalytic activity for oxygen electro-reduction. J Power Sources, 2012, 197 : $107-110$

Open Access This article is distributed under the terms of the Creative Commons Attribution License which permits any use, distribution, and reproduction in any medium, provided the original author(s) and source are credited. 